\title{
Mobility enhancement among older adults $75+$ in rural areas: Study protocol of the MOBILE randomized controlled trial
}

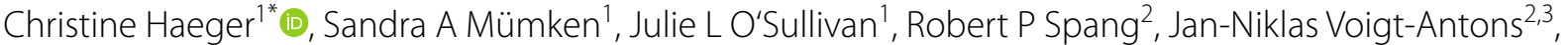 \\ Martin Stockburger ${ }^{1,4}$, Dagmar Dräger ${ }^{1}$ and Paul Gellert ${ }^{1}$
}

\begin{abstract}
Background: Maintaining mobility in old age is crucial for healthy ageing including delaying the onset and progress of frailty. However, the extent of an individuals' mobility relies largely on their personal, social, and environmental resources as outlined in the Life-Space Constriction Model. Recent studies mainly focus on facilitating habitual outof-home mobility by fostering one type of resources only. The MOBILE trial aims at testing whether tablet-assisted motivational counselling enhances the mobility of community-dwelling older adults by addressing personal, social, and environmental resources.
\end{abstract}

Methods: In the MOBILE randomized controlled trial, we plan to enrol 254 community-dwelling older adults aged 75 and older from Havelland, a rural area in Germany. The intervention group will receive a tablet-assisted motivational counselling at the participant's home and two follow-up telephone sessions. Main focus of the counselling sessions lays on setting and adapting individual mobility goals and applying action planning and habit formation strategies by incorporating the personal social network and regional opportunities for engaging in mobility related activities. The control group will receive postal general health information. The primary mobility outcome is time out-of-home assessed by GPS (GPS.Rec2.0-App) at three points in time (baseline, after one month, and after three months for seven consecutive days each). Secondary outcomes are the size of the GPS-derived life-space convex hull, self-reported life-space mobility (LSA-D), physical activity (IPAQ), depressive symptoms (GDS), frailty phenotype, and health status (SF-12).

Discussion: The MOBILE trial will test the effect of a motivational counselling intervention on out-of-home mobility in community-dwelling older adults. Novel aspects of the MOBILE trial include the preventive multi-level intervention approach in combination with easy-to-use technology. The ecological approach ensures low-threshold implementation, which increases the benefit for the people in the region.

Trial registration: The MOBILE trial is prospectively registered at DRKS (Deutsches Register Klinischer Studien, German Registry of Clinical Trials) DRKS00025230. Registered 5 May 2021.

Keywords: Rural areas, Mobility in old age, Preventive home visit, Globalpositioning system (GPS)

*Correspondence: christine.haeger@charite.de

${ }^{1}$ Charité Universitätsmedizin Berlin corporate member of Freie Universität Berlin Humboldt Universität zu Berlin, Institute of Medical Sociology

Rehabilitation Science, Charitéplatz 1, 10117 Berlin, Germany

Full list of author information is available at the end of the article

\section{Background}

Due to current and future changes in demographic society distributions, more attention must be paid to the aspect of healthy ageing [1]. Healthy ageing is challenged as people get older is the growing prevalence of 
the frailty syndrome that varies from 4 to $59 \%$ depending on the used conceptualisation, assessments, and defined age group [2]. Frailty is characterised by reduced physical reserves, activity restrictions, and a low-level of social participation leading to a decreased ability to cope with everyday stressors, a higher risk of adverse health outcomes, and overall reduced mobility [3, 4]. Therefore, interventions to prevent or delay the onset of frailty that are easy to apply and scalable are crucially needed. One way of preventing or delaying frailty is the facilitation of out-of-home mobility [5], as it is a prerequisite for participation in physical activities, social in-person involvement, and access to health care [6, 7]. Out-of-home mobility can be can be defined as movement through outdoor living environments called life-spaces expanding from one's home to the neighbourhood, city lived in, and beyond $[7,8]$.

According to the Life-Space Constriction model, a person's out-of-home mobility is determined by a combination of personal (e.g., health status, health literacy, coping strategies), social (e.g., social network, connection to neighbourhood), and environmental (e.g., regional infrastructure, access to health care) resources and obstacles [9], indicating that multi-level interventions on facilitating out-of-home-mobility seem to be most promising [5, 9]. Although the Life-Space Constriction model proposes the combination of individual, social and environmental resources and although there is evidence from longitudinal studies on the relationship between social factors and mobility [10] most interventional studies largely relied on addressing personal resources only. Personal goal setting and motivational support seem to be promising components as highlighted in the AGNES trial [11]. This applies especially when used techniques are based on well-founded psychological theories such as coping with loss or onset of disability as well as self-regulatory strategies to change behaviour $[12,13]$. There is little evidence about the use of behaviour change techniques to maintain or modify out-of-home mobility in interventions to prevent frailty and one promising but solemnly used technique may be the formation of routines/habits $[14,15]$. In addition results of a recent intervention study in Finland suggest that more evidence is needed on the short- and long-term effects of face-to-face counselling interventions addressing participation in mobility-related activities [16]. However, in the Finnish study, out-ofhome mobility was solely measured using self-reported instruments. There is a growing body of evidence that out-of-home mobility is measured more precisely using Global Positioning Systems (GPS) data rather than the commonly used self-reported questionnaires [17]. Additionally there is existing evidence that out-of-home mobility is notably restricted in those older adults living in rural areas due to less infrastructure, car-dependency, and difficult access to health care, indicating the need of intervention studies in rural areas [18]. Hence, little research has been done when it comes to combining individual goal setting with social and environmental resources in a multi-component intervention with objective GPS measurements, especially when focusing on prevention or reversing frailty in older adults in rural areas $[5,19,20]$. To close the defined research gaps, the MOBILE (Mobility in Old age By Integrating health care and personal network resources in older adults Living in rural arEas) trial is aiming to determine whether a motivational counselling intervention can facilitate out-ofhome mobility in older adults living in rural areas.

\section{Objectives}

The main objective of the MOBILE trial is to test the effectiveness of a motivational counselling intervention to improve out-of-home mobility and thereby possibly preventing or delaying frailty in community-dwelling older adults in a particular rural area. Furthermore, drawing on the Life-Space Constriction model, we will be investigating the resources at personal, social, and environmental level and their relationship with out-of-home mobility, which, in turn, will be related with indicators of health, wellbeing, and frailty.

\section{Methods/design}

\section{Trial design}

The MOBILE trial is a randomized controlled interventional study using a pragmatic sample comparing an intervention to facilitate out-of-home mobility with generic health and mobility information that apply to the participants living area. Data will be collected as Computer Assisted Personal Interview (CAPI) and Computer Assisted Telephone Interviews (CATI) on five points in time $\left(\mathrm{T}_{0}-\mathrm{T}_{4}\right)$ and will consist of questionnaires, assessments, and objective GPS measurements. At baseline $\left(\mathrm{T}_{0}\right)$ a comprehensive questionnaire consisting of established valid scales focusing on personal, social, and environmental resources is conducted as CAPI by a trained study nurse. Furthermore, physical assessments (i.e., mobility and walking test, handgrip) will be included at baseline. $T_{1}$ is planned as a short questionnaire after the first intervention session including change-sensitive scales to capture immediate intervention effects, whereas $\mathrm{T}_{2}$ will serve as a follow-up measurement where most parts of the baseline questionnaire (excluding sociodemographic variable) are repeated. $\mathrm{T}_{3}$ and $\mathrm{T}_{4}$ are short telephone-based interviews with focus on personal resources. An overview of the used scales and assessments at each measuring point is outlined in Table 1. 
Table 1 Outcome Measure of the MOBILE trial

\begin{tabular}{|c|c|c|c|c|c|c|c|}
\hline \multirow{2}{*}{$\begin{array}{l}\text { Outcome Measure } \\
\text { Main Outcome }\end{array}$} & \multirow[t]{2}{*}{ Operationalisation (Type of assessment) } & \multicolumn{5}{|c|}{ Times of assessment } & \multirow[t]{2}{*}{ Ref } \\
\hline & & $\mathrm{T}_{0}$ & $\mathrm{~T}_{1}$ & $T_{2}$ & $\mathrm{~T}_{3}$ & $\mathrm{~T}_{4}$ & \\
\hline Time out-of-home & GPS tracker for seven consecutive days & $S$ & S & $\mathrm{S}$ & - & - & [25] \\
\hline \multicolumn{8}{|l|}{ Secondary Outcomes } \\
\hline Life-Space parameter & Aggregated GPS data (i.e. convex hull) & $\mathrm{S}$ & S & $\mathrm{S}$ & - & - & [25] \\
\hline Self-perceived life-space & LSA-D & $\mathrm{H}$ & $\mathrm{H}$ & $\mathrm{H}$ & $\mathrm{T}$ & $\mathrm{T}$ & {$[6]$} \\
\hline Physical activity & IPAQ & $\mathrm{H}$ & $\mathrm{H}$ & $\mathrm{H}$ & - & - & {$[29]$} \\
\hline Depressive symptoms & GDS-12R & $\mathrm{H}$ & - & $\mathrm{H}$ & - & - & [30] \\
\hline Health Status & SF-12 & $\mathrm{H}$ & $\mathrm{H}$ & $\mathrm{H}$ & - & $\mathrm{T}$ & [32] \\
\hline Frailty & Frailty-phenotype Fried & $\mathrm{H}$ & - & $\mathrm{H}$ & - & $\mathrm{T}$ & [34] \\
\hline \multicolumn{8}{|l|}{ Mediator variables } \\
\hline Goal attainment & Selected items from SOC-questionnaires & $\mathrm{H}$ & $\mathrm{H}$ & $\mathrm{H}$ & - & - & [46] \\
\hline Habits & 4 items from SRHI & $\mathrm{H}$ & $\mathrm{H}$ & $\mathrm{H}$ & - & - & [47] \\
\hline Lifestyle chance & $\begin{array}{l}\text { Action and coping planning, adapted } \\
\text { from Schwarzer et al. } 2008\end{array}$ & $\mathrm{H}$ & $\mathrm{H}$ & $\mathrm{H}$ & - & - & {$[48]$} \\
\hline Balance confidence & $A B C-6$ & $\mathrm{H}$ & - & $\mathrm{H}$ & - & - & [49] \\
\hline Health literacy & HLS-EU-Q16 & $\mathrm{H}$ & - & $\mathrm{H}$ & $T$ & $\mathrm{~T}$ & [50] \\
\hline Views on ageing & Adapted from DEAS survey & $\mathrm{H}$ & - & $\mathrm{H}$ & - & - & [51] \\
\hline Connection to place & $\begin{array}{l}\text { Selected items from housing enabler } \\
\text { questionnaires }\end{array}$ & $\mathrm{H}$ & - & - & - & - & {$[52]$} \\
\hline Access to health care & Own items & $\mathrm{H}$ & - & - & - & - & - \\
\hline Utilization of health care & Own items & $\mathrm{H}$ & - & - & - & - & - \\
\hline Connection to neighbourhood & Selected items from DEAS survey & $\mathrm{H}$ & - & - & - & - & [53] \\
\hline Social network & Adapted from SHARE survey & $\mathrm{H}$ & - & $\mathrm{H}$ & - & - & \\
\hline Loneliness & UCLA Three-Item Loneliness Scale & $\mathrm{H}$ & - & $\mathrm{H}$ & - & - & [54] \\
\hline Social Participation & Own items, adapted from SHARE survey & $\mathrm{H}$ & - & $\mathrm{H}$ & $\mathrm{T}$ & $\mathrm{T}$ & [51] \\
\hline \multicolumn{8}{|l|}{ Covariates } \\
\hline Sociodemographic data & $\begin{array}{l}\text { Age, gender education level, level of care, } \\
\text { living arrangements }\end{array}$ & $\mathrm{H}$ & - & - & - & - & - \\
\hline Physical performance & DEMMI & $\mathrm{H}$ & - & $\mathrm{H}$ & - & - & [55] \\
\hline Hand grip & $\begin{array}{l}\text { Gauged Hand grip dynamometer, } \\
\text { Sahean SH5001 }\end{array}$ & $\mathrm{H}$ & - & $\mathrm{H}$ & - & - & - \\
\hline History of falls & Single-item question & $\mathrm{H}$ & $\mathrm{H}$ & $\mathrm{H}$ & $\mathrm{T}$ & $\mathrm{T}$ & - \\
\hline Utilization of assistive devices & Own questions, adapted from Baker, 2003 & $\mathrm{H}$ & - & - & - & - & {$[6]$} \\
\hline Cognition & Mini-Cog & $\mathrm{H}$ & - & - & - & - & [56] \\
\hline Corona & $\begin{array}{l}\text { Covid-19 history, vaccination status, } \\
\text { anxiety, impact on daily life } \\
\text { (own items, single-questions) }\end{array}$ & $\mathrm{H}$ & - & - & - & - & - \\
\hline $\begin{array}{l}\text { Living surroundings, observa- } \\
\text { tion by interviewer }\end{array}$ & $\begin{array}{l}\text { Type of house, stairs in front or within } \\
\text { the building, pavement }\end{array}$ & $\mathrm{H}$ & - & - & - & - & - \\
\hline
\end{tabular}

$H$, Home based interview CAPI, $S$ self-assessment of GPS-Data with study mobile phone, $T$ Telephone-based interview CATI, $T_{0}$ Baseline measurement, $T_{1}$ one months after baseline, $T_{2}$ three months after baseline, $T_{3} 6$ months after baseline, $T_{4} 12$ months after baseline, Ref. reference, GPS Global Positioning System, $L S A-D$ German version of the University of Alabama Life-Space Assessment, IPAQ International Physical Activity Questionnaire, GDS-12R short residential Version of the Geriatric Depression Scale, SF-12 The Short Form Health Survey, SOC Selection, Optimization, and Compensation, SRHI Self-Report Index of Habit Strength, ABC-6 Activityspecific Balance Confidence scale HLS-EU-Q16 Health Literacy Scale, 16 items, DEAS German Ageing Study / Deutscher Alterssurvey, SHARE The Survey of Health, Ageing and Retirement in Europe, DEMMI De Morton Mobility Index, Mini-Cog Screening for Cognitive Impairment in Older Adults

All participants will be equipped with needed technology to track the GPS coordinates and activity diaries measuring out-of-home mobility at three points in time (as outlined in Fig. 1 ) for seven consecutive days each. GPS data is measured using a study mobile phone (ZTE Blade A5) with the "GPS.Rec2.0"-App as the only working application. The app was developed solely for the purpose of this trial and was tested and adapted as part of piloting work. "GPS.Rec2.0" is a mere data collection app and there is no interaction with the study participant. GPS location is recorded every ten seconds with an accuracy of up to $25 \mathrm{~m}$. A detailed description and availability on the technical development of the "GPS.Rec2.0" application is going to be published elsewhere.

The participants in the intervention condition will receive motivational counselling (i.e., one home visit and up to two phone calls) from a trained physiotherapist with the focus of individual mobility goals. Participants 


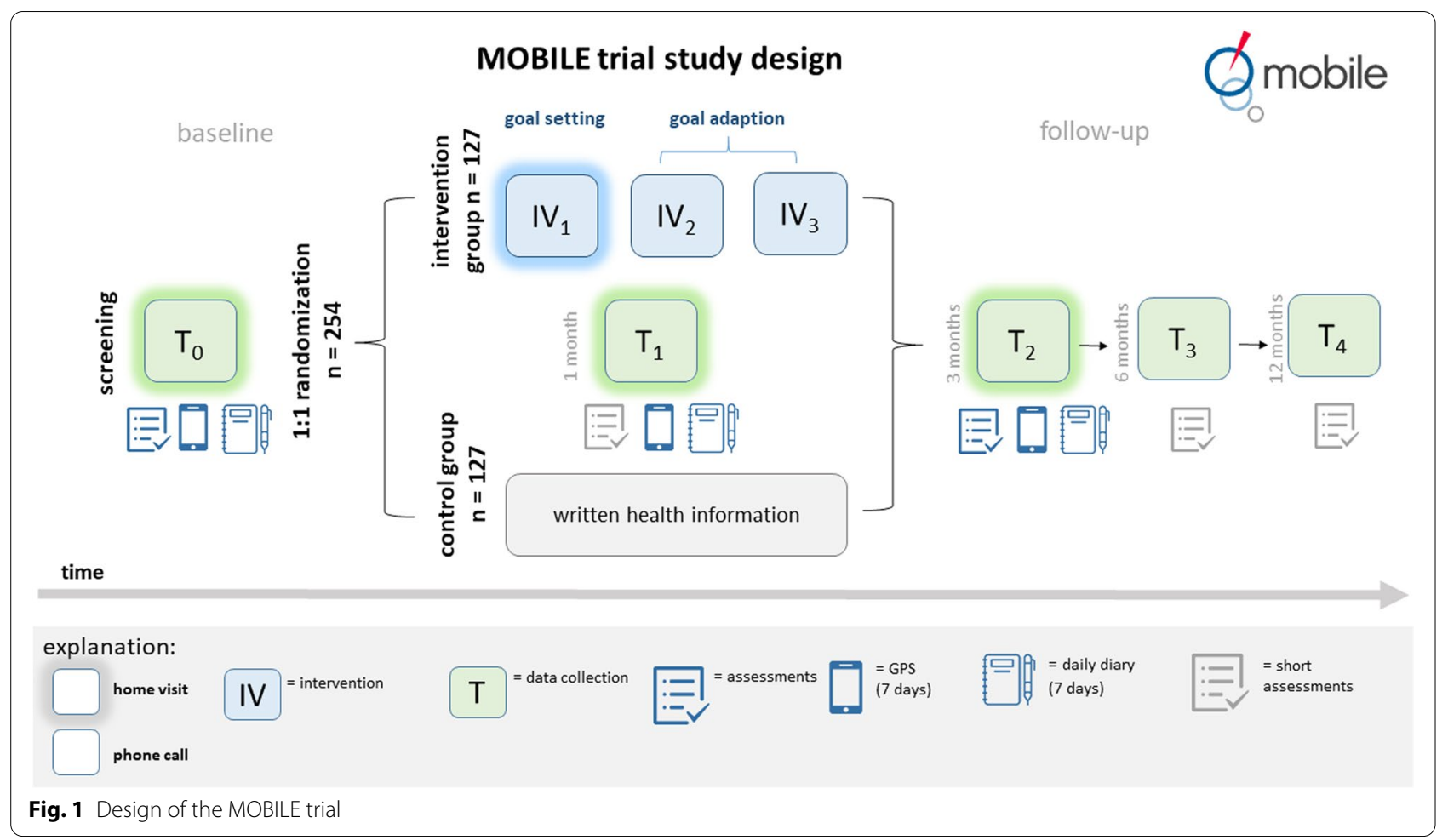

in the control condition will be receiving written information on health and mobility.

\section{Adaptions due to Corona-pandemic}

As the Covid-19 pandemic is both unpredictable and dynamic, changes in the execution of the intervention and questionnaires at $\mathrm{T}_{0}, \mathrm{~T}_{1}$, and $\mathrm{T}_{2}$ may become necessary. If the situation requires it, we will switch to remote option for the aforementioned tasks. To minimize the risk of infections in general, all contacts with participants will take place in compliance with hygiene regulations of Charité - Universitätsmedizin Berlin and federal state of Brandenburg. Key components of the MOBILE hygiene concept include full vaccination of study personal, wearing FFP2-masks, sanitizing, and ventilation at scheduled intervals. Although protocol deviations may occur due to the pandemic, the main components of the trial, i.e., assessment of study variables and delivery of the intervention will still be feasible, albeit with adjustments, such as telephone instead of personal interviews.

\section{Participants, interventions, and outcomes Study setting}

The MOBILE trial will be conducted in the Havelland region, a rural area in Brandenburg, in the eastern part of Germany next to Berlin, Germany. The region Havelland is characterized by mainly flat landscape, vast areas of forest land, stretches of water, and farming, and a generally low population-density with about 150,000 inhabitants. The study centre is located at the Institute of Medical Sociology and Rehabilitation Science, Charité Universitätsmedizin Berlin.

\section{Eligibility criteria}

Participants will be included, if they fulfil all inclusion criteria and none of the exclusion criteria, which are as follows. Inclusion criteria are:

- living in region Havelland, Brandenburg, Germany,

- 75 years or older

- community-dwelling

- having sufficient mobility (i.e., being able to move autonomously or with walking devices and/or little help of others),

- ability tfo give informed consent

- are willing to participate. Exclusion criteria are:

- severe cognitive impairments, that prevent living independently

- acute severe events within the past 4 weeks (e.g., falls, operation), that influence out-of-home mobility

- insufficient understanding of German language, 
- severe impairment in vision or hearing that will avert participant's ability to follow the instructions of the study personnel

- reasons of exclusion will be recorded and reported.

\section{Intervention}

The intervention is designed as a tablet-assisted motivational counselling intervention to enhance out-of-home mobility. Summarized it consists of one goal setting and two goal adaption sessions delivered by either one of the two physiotherapists of the study group. All sessions are complemented by the "MOBILE-Intervention-App" (iOS) which is operated by the physiotherapist and works as a documentation and visualization tool. The app was developed by our study team to assist needs of the intervention performance and screenshots of the three main screens of the MOBILE-Intervention-App are depicted in Fig. 2. Main goal of this intervention is to set and adapt individual goals (e.g., going for a walk, participating in group activity) to foster out-of-home mobility of the participant. All sessions are delivered by the same physiotherapist to rule out information deficits. Couples can take part in the study and are allocated in the same group with each partner receiving separate assessments $\left(T_{0}-T_{5}\right)$. Regarding the intervention, however, there is no strict protocol and couples can either participate together or separately according to their personal preferences and needs. Even when a couple is choosing a joint intervention, mobility goals can still be personalised for each partner and individual profiles are generated in either way. Details of the intervention sessions are described in the following sections and are further classified according to the Behavioural Change Technique Taxonomy (BCT v1) [21]. The BCT Taxonomy was developed by Michie et al. and can be used as a guidance for comparable reporting of complex behavioural interventions. We expect no harms of the intervention and there will be debriefing after the trial.

\section{Goal setting (IV $)$}

After the introduction, overview of the study scope, and relationship establishment, the importance of mobility in old age in terms of social, physical, and mental benefits will be highlighted by the physiotherapist (BCT: information about health consequences 5.1, information about social and environmental consequences 5.3; see [21]). Both physiotherapists are trained on activating positive und relativizing negative views on ageing and mobility by reframing arising negative automatic thoughts and by representing a general age-appreciating attitude (framing/reframing 13.2) [22, 23]. The training consisted of extensive literature research, peer-to-peer counselling, and development of a "learning guide". The latter is composed of a handbook that will help guiding through the sessions and will be amplified and adjusted by the study group during the scope of intervention.

The main objective of the first session is the setting of individual mobility goals (goal setting (behaviour) 1.1, goal setting (outcome) 1.3) in relation with social network analysis (social support (practical) 3.2, social support (emotional) 3.3) and linking regional services and opportunities for mobility (social support (unspecified) 3.1, action planning 1.4). This will be done by firstly filling out the personal network map (Screen 2) to illustrate existing resources. In a second step, a regional network map with existing opportunities for activity engagement is demonstrated (Screen 3) to point out environmental/ social resources and link them with the planned goals (Screen 1). The database underlying the regional network map was generated by the study group prior enrolment and is updated on a regular basis. After illustrating both personal and environmental/social resources, individual mobility goals are discussed and recorded in the intervention app (Screen 1). It is planned to intended at least two distinct mobility goals: One of them being a routine goal (e.g., going for a walk every Monday morning) (action planning 1.4, habit formation 8.3) and the other one being a goal with a specific destination (e.g., going to a museum, action planning 1.4) that is asked to be routine as well, yet this is not mandatory. It is important to note that a defined goal can also be maintaining the current mobility status (focus on past success 15.3), as maintaining mobility is important for prevention of frailty despite possible decline in physical capacities in old age. Any foreseeable barrier that may impede goal achievement will also be discussed (e.g., loss of physical capacity, pain, environmental challenges) (action planning 1.4). Goal selection and strategies to attain the goals will be set and explored based on the assumption of the selection (goal setting (behaviour) 1.1, goal setting (outcome) 1.3, action planning 1.4), optimization (commitment 1.9), and compensation (coping planning 1.2) (SOC) approach [24]. All screens can be printed out directly during the session by using a battery-printer with internal Wi-Fi to ensure that printing works everywhere (i.e., Canon Pixma TR150). This will enhance recognition of the first session, as the participants can keep record of the conversation. The first session will take approximately 60 to $90 \mathrm{~min}$ and a new appointment for the following session will be scheduled individually within four weeks.

\section{Goal adaption ( $I V_{2}$ and $\left.I V_{3}\right)$}

The booster sessions will be performed as telephone interviews and focus on goal adaption and habit formation. The foundation of these sessions are the defined 


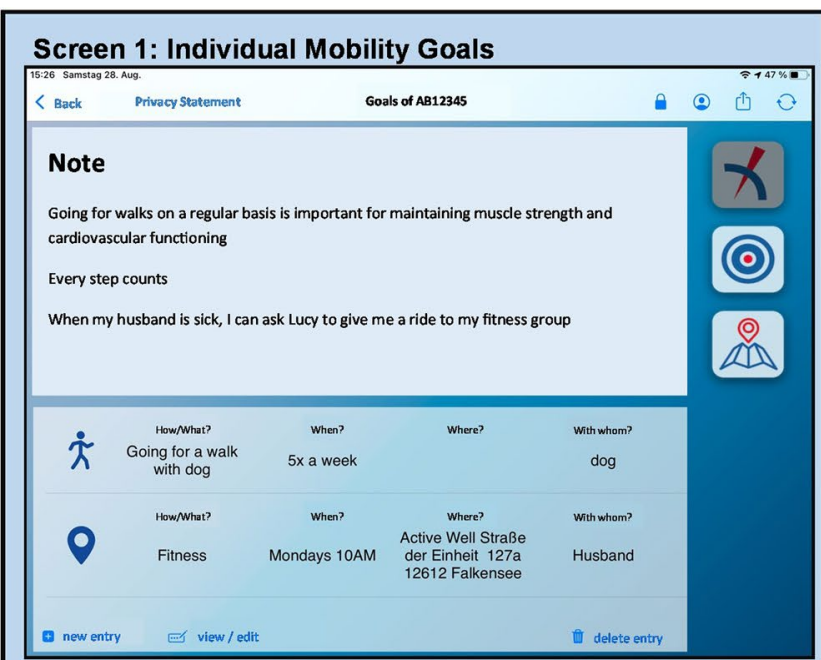

Screen 2: Personal network

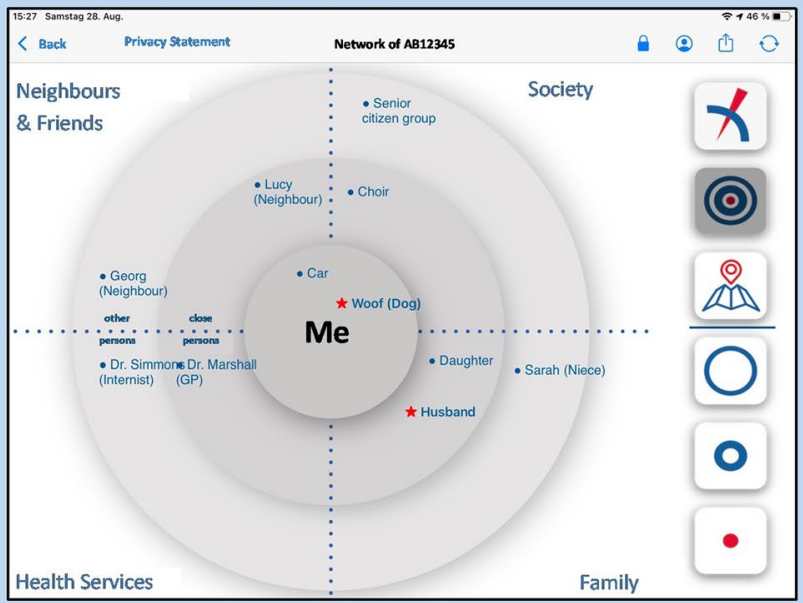

Screen 3: Regional map with activity opportunities

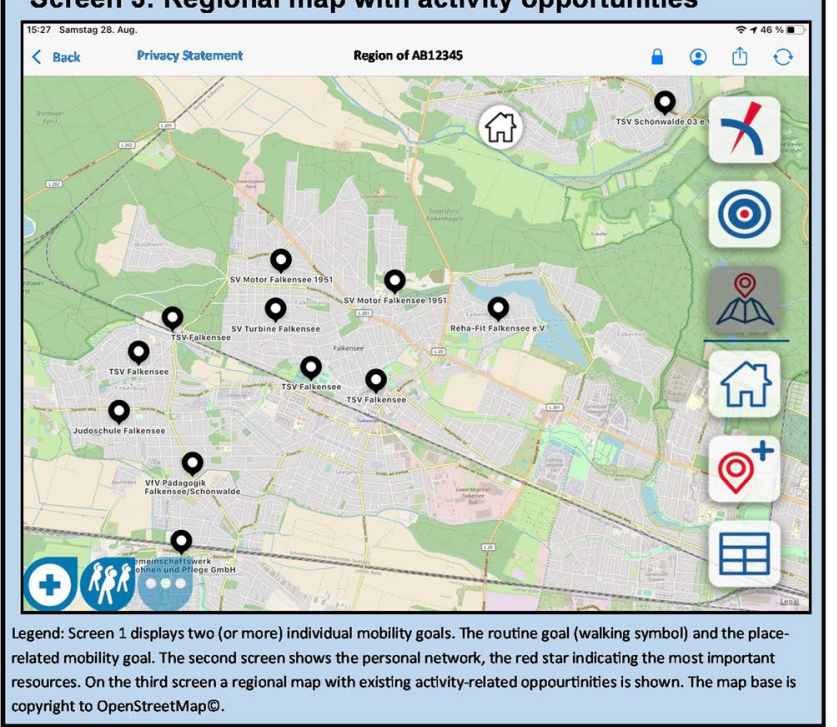

Fig. 2 Outline of the Mobile-Intervention-App 
mobility goals that have been established in the first interview. For easy and simple guidance, the outlines of the personal network map and mobility goals that were printed out at the first intervention session are used. The personal network map and each goal will be individually reviewed and discussed regarding whether it was well chosen or if any unexpected barriers occurred (review behaviour goal(s) 1.5, feedback on behaviour 2.2). Goal adaption will then be individually and flexibly adjusted to personal circumstances (action planning 1.4, coping planning 1.2). Moreover, these sessions should not only focus on barriers or optimization but also affirm and encourage (focus on past success 15.3) on goals that worked well, especially when the goal is maintaining the current mobility status. A further focus of the follow-up sessions is to transfer the chosen goals into habitual routines of out-of-home mobility (8.3 habit formation). A documentation of the goal adaption session is sent to the person's home if major modifications have been made. Time needed for each goal adaption session will be about 30 to $45 \mathrm{~min}$.

\section{Control condition}

The control condition will receive a booklet containing general health information (information about antecedents 4.2, information about health consequences 5.1) and advice on how to get more active (social support (unspecified) 4.1). This booklet contains eight pages in A5 format and besides general advice on healthy ageing also includes a page featuring local stakeholder that offer mobility-related (group) activities and one page for phrasing individual goals (goal setting (behaviour) 1.1).

Critical events will be reported for both intervention and control condition. In order to ensure participation adherence the study team contacts participants via phone on a regular basis.

\section{Outcomes}

\section{Primary outcome}

The primary outcome variable is "time out-of-home" $(\mathrm{TOH})$ based on the objective GPS measurement and meaning the total time spent outside of one's home radius (i.e. $50 \mathrm{~m}$ ) per day. $\mathrm{TOH}$ can be measured in minutes or percentage. A day in this connection is defined from 3:00 AM to 02:59 AM to include trips than last longer than midnight and as recommended in literature $[25,26]$. GPS data is monitored on seven consecutive days at $\mathrm{T}_{0}, \mathrm{~T}_{1}$, and $\mathrm{T}_{2}$ respectively. An overview of all outcomes, assessments and time of measurement is demonstrated in Table 1.

\section{Secondary outcomes}

Aggregated GPS data: Raw GPS data can be aggregated to certain parameters to describe someone's life-space. Here we use "convex hull", a descriptive measure to specify the area of all daily GPS fixes [25]. We will further acquire other variables (such as time first moved, standard deviational ellipse, maximum distance of home) that have been identified as important in literature [7, 25, 27]

Self-perceived life-space mobility: This is measured using the German version of the University of Alabama Life-Space Assessment [8], that has been translated and validated for the use in urban and rural settings by our study group prior to the MOBILE trial [28]. The lifespace is assessed both at times of assessment $\left(\mathrm{T}_{0}, \mathrm{~T}_{1}\right.$, and $\mathrm{T}_{2}$ ) on a weekly basis and during the seven-day GPS tracking on a daily basis as part of the activity diary.

Physical activity: Physical activity is assessed using the International Physical Activity Questionnaire (IPAQ) in the short version for older adults [29]. This short version consists of four questions addressing both the number of times per week and minutes per days of strenuous activities, moderate activities, walking, and sitting. A score is then generated and the categories of high, moderate, and low level of activity are composed.

Depressive symptoms: Depressive symptoms are addressed using a validated shorter version [30] of the Geriatric Depression Scale [31]. In the shorter version for residential study population, 12 dichotomous questions are asked and a score is then evaluated.

Health status: The international approved Short-Form 12 (SF-12) [32] of the 36-item short-form health survey (SF-36) [33] is used to measure the subjective overall health status for the last seven days.

Operationalisation of Frailty: The used Frailty phenotype approach is based on a cluster of five clinical criteria (reduced muscle strength, slow gait speed, exhaustion, weight loss, low level of physical activity) developed by Fried et al. in 2001 [34]. This phenotype is one of the most popular tools to evaluate frailty, predict adverse health outcomes and distinguishes well between three different stages of frailty (robust, pre-frail, frail) in older adult [34, 35]. To rate the physical criteria of the phenotype, functional tests are performed using the SAHEAN 5001 hand grip dynamometer to assess handgrip strength [34] and a $3 \mathrm{~m}$ walking test in preferred speed to measure gait speed [36]. To assess the criterion of exhaustion two questions of the Centre for Epidemiological Studies depression (CES-D) scale are used like in the original publication by Fried et al. and weight loss was defined as unintentional weight loss of $\geq 5 \mathrm{KG}$ in the prior year [34]. The criterion of low physical activity is retrieved 
from a single item question of the validated SHAREfrailty phenotype Instrument [37].

Mediators and covariates are also outlined in Table 1 and contain personal, social, and environmental resources.

\section{Sample size}

For the sample size calculation, results of previous intervention studies for increasing mobility in older adults were considered [38, 39]. A relevant change in mobility is considered as an increase in mobility of $15 \%$ after three months (Life Space $M=65.4 \quad(S D=20.1)+15 \%=75.2$ [40] in comparison to the control condition (1:1 ratio, power 1 - beta $=95 \%$, alpha (two-sided $)=5 \%$, dropout $=15 \%)$. Based on these presumptions an online tool (powerandsamplesize.com) has been used to calculate sample size, which is stated as follows: $N=110+15 \%$ dropout in each condition and therefore a total sample size of $N=254$ ( $N=127$ in each condition $)$ is needed.

\section{Recruitment of participants}

A number of complementary strategies will be employed for the recruitment of participants. First approaches include a newspaper article, displaying of study information flyer on designated spots (e.g., supermarkets, pharmacies, health or public institution), and recruitment via our stakeholder network. Further steps will include postal invitations to all citizen of Havelland aged 75 and older (data has been provided by the public register for the purpose of this trial), presentation of our trial on various occasions (e.g., sport groups, social activities, public events), internet magazine articles, and advertisement. We implemented a website, a flyer, and posters to complement our recruitment strategy. Recruitment success of each path and strategy will be tracked and readjusted if applicable. Once an interested person contacts the MOBILE study team, the initial screening for eligibility (age, place of living, mobility) will be performed via phone, where questions can be asked and the further course of the study will be explained. Those, who are interested and eligible, will then receive the study information letter, consent forms, and hygienic concept via post. Informed consent form will be collected at the baseline measurement $\left(\mathrm{T}_{0}\right)$ once the initial screening is confirmed by the study nurse. Study participants receive no financial compensation, however all participants get to keep the bumbag with the study emblem printed on, that was given to them with the GPS-tracker.

\section{Assignment of Interventions Allocation and blinding}

After baseline assessment, participants included in the study will be randomly assigned to intervention or control condition. Due to practical implications a couple will always be assigned to the same group. Randomization to either intervention or control condition is performed computer-assisted with 1:1 ratio by using the package "blockrand" (block randomization with block sizes of 4,6 , and 8 as well as strata) using $R$ version 4.0.4. Strata are as follows: older (>80 years) / younger (75 80 years) and living in the centre of one of the three biggest towns in Havelland (i.e. 15.000 inhabitants) / not living in the centre of one of these three biggest towns). The centre of every single town was defined individually by criteria of buildings in closed development, provided services, and infrastructure [41].

Allocation is concealed until after baseline measurement, when the study nurse uncovers the allocation. Randomization sequence, enrolment of participants, and allocation of the participants are strictly separated. The study nurse and participants are blinded during the baseline measurement. Since our proposed trial includes a home visit (in comparison to a written health information) blinding for study personal or participants cannot fully be maintained over the further course of the study. GPS data will be processed and analysed by data scientists at TU Berlin who will be blinded throughout the whole study period.

\section{Data collection, management and statistical analysis Data Collection}

All data is collecting using a Charité-owned laptop and data is recorded in a Microsoft Access 2016 database to ensure easy exporting to SPSS v25 (IBM, Cary, Ind.) where statistical analysis will be primarily performed. GPS data of the participants is stored on the study mobile phone and is then uploaded by the study team on Charité premises to the TU Berlin server to ensure secure data handling.

\section{Data management}

The collected data is regularly checked upon consistency, accuracy, and completeness. Since the data is solely collected digitally (except activity diary) an automated backup system (once a week) has been implemented. GPS data is also regularly reviewed for accordance with the activity diary. Only the study team will have access to the data, as defined in the data protection concept that was approved by ethics committee and clinical trial office.

\section{Statistical analysis}

Raw GPS data will be checked and aggregated by a computer scientist of the TU Berlin und will be prepared for further analyses. All analyses will be performed using SPSS v25 (IBM, Cary, Inc.) and in R Project using the intention-to-treat principle. For the demographic 
variables, means, standard deviations and frequencies will be calculated, and distribution will be checked. Medians and interquartile ranges will be reported for non-normally distributed data. To test the primary hypothesis, multilevel modelling will be used to estimate the group difference in the primary outcome across $\mathrm{T}_{0}-\mathrm{T}_{2}$ as predefined in the power analysis. Covariance analysis will also examine possible mediating variables declaring any relationship between out-of-home mobility and individual resources. The level of significance will be set to $\mathrm{p}<0.05$ (two-sided) and $95 \%$ confidence interval will be reported. Multiple imputations using a package (e.g., AMELIA II) will be applied to handle missing data if necessary.

\section{Trial status}

Rolling recruitment started on 7 June 2021 (i.e., first participant in) for a duration of presumably 12 months, meaning that the last participants included in the study will have their baseline assessment in June 2022 und their last assessment one year later in June 2023 marking the end of the field phase. Recruitment can be extended by up to six months, if the required number of participants has not yet been reached. The funding period for this trial is until February 2024.

\section{Ethics and dissemination \\ Research ethics approval}

This trial was approved by the Ethics Committee of Charité - Universitätsmedizin Berlin (14/05/2020, EA1/052/20).

\section{Protocol amendments}

Relevant changes of protocol will be stated in the trial paper.

\section{Consent or assent}

The participant of the MOBILE trial will receive comprehensive information on the research project and trial and written informed consent will be obtained by study nurse prior to data collection.

\section{Declaration of interests}

The authors declare that they have no competing interests.

\section{Access to data}

Data collected during the study will be available under the search term "MOBILE" at the institutional refubium repositorium after study is completed and in an aggregated and anonymized form, whereas anonymity cannot be guaranteed at a raw data level.

\section{Dissemination policy}

Publication of trial results are planned to be published in peer-reviewed journals. As for public audience results will be disseminated through low-threshold channels such as local talks, newspaper articles or interviews. Both codes of the developed apps ("GPS.Rec.2.0" and "MOBILE-Intervention-App") are planned to be available as open source.

\section{Discussion}

The proposed MOBILE trial will advance the knowledge on the effects of a motivational counselling for fostering out-of-home mobility in community-dwelling older adults in rural areas. Furthermore, we will achieve a deeper understanding of underlying individual, social and environmental resources for out-of-home mobility and their interconnection in this population. Approaches that at least focusing on the individual resources have been tested in Finland (i.e. COSMOS Trial, AGNES Study) [11, 16, 42], and are currently under investigation [42]. However, these trials specifically looked into physical activity, falls, and healthy aging in general and therefore have merely incorporated self-reported measurements of out-of-home mobility. In contrast, the main outcome of the proposed MOBILE Trial is out-of-home mobility based on GPS data, which are to become state-of-the-art of measuring mobility [17, 43]. This includes derived specific mobility indicators (e.g., time spent out-of-home, maximal distance from home, convex hull) $[25,44,45]$ and the linkage of GPS and movement data with geographic information systems (GIS). Another notable benefit of objective mobility measure is that we can match participant's individual goals with their real performance within the interval of assessment. As the out-of-home mobility is largely represented by moving through different life-spaces we use the Life-Space Assessment [8] and validated the instrument for the German population [28]. The ecological approach assures high accessibility and easy implementation, thus maximizing the benefits for the people within the region.

\footnotetext{
Abbreviations

ABC: Activities-specific Balance Confidence scale; BCT: Behavioral Change Technique; BMBF: Bundesministerium für Bildung und Forschung (Federal Ministry of Research and Education); CAPI: Computer-assisted personal interview; CATI: Computer-assisted telephone interview; DEAS: Deutscher Alterssurvey (German Ageing Survey); DEMMI: De Morton Mobility Index; DRKS:: Deutsches Register Klinischer Studien (German Registry of Clinical Trials); GDS: Geriatric Depression Scale; GPS: Global Positioning System; GIS: Geographic Information System; HLS: Health Literacy Scale; IPAQ: International
} 
Physical Activity Questionnaire; LSA-D: German Version of Life-Space Assessment; SHARE: The Survey of Health, Ageing and Retirement in Europe; SF-12: The Short Form Health Survey; SOC: Selection, Optimization and Compensation; SRHI: Self-Reported Habit Index; TOH: Time out-of-home; TU Berlin: Technische Universität Berlin.

\section{Acknowledgements}

We acknowledge support from Lan Thao Nguyen and Arik Grahl for the development of the MOBILE technology setup and Malte Stollwerck and Ruth Priesching for supporting study execution. We also acknowledge support from Stephan Dombrowski for advising the use of the BCT taxonomy.

\section{Authors' contributions}

PG conceptualized the study. $\mathrm{CH}$ developed the first draft. All authors ( $\mathrm{CH}, \mathrm{SM}$, $J O, R S, J N V A, M S, D D, P G)$ made substantial suggestions to further qualify the first draft. All authors approved the final manuscript.

\section{Funding}

Open Access funding enabled and organized by Projekt DEAL. This trial is funded by the BMBF (Federal Ministry of Research and Education, ID: 01GY1803). There is no interfering in the design of the study nor in execution, analyses, interpretation of data or submission of the results on behalf of the funder. JNVA and RS are partially funded under the JTC 2017 launched by the Joint Programming Initiative "More Years Better Lives" (JPI MYBL), which is supported by Horizon2020, the EU Framework for Research and Innovation (Grant Agreement nr 643850) and by the Innovation Fund of the Federal Joint Committee (G-BA), (Grant Project "DemTab" Grant-Number 01VSF17039). Open access funding provided by Project DEAL. Bundesministerium für Bildung und Forschung,01GY1803,Paul Gellert

\section{Availability of data and materials}

Data collected during the study will be available upon reasonable request

\section{Declarations}

\section{Ethics approval and consent to participate}

This research was approved by the Ethics Committee of Charité - Universitätsmedizin Berlin 14/05/2020; EA1/052/20). All participants will give written informed consent before included in the trial. The trial was prospectively registered at the DRKS registry on 5 May 2021 (Deutsches Register Klinischer Studien) with the study identity number DRKS00025230. https://www.drks.de/ drks_web/navigate.do? navigationld=trial.HTML\&TRIAL_ID=DRKS00025230

\section{Consent for publication}

Not applicable as this protocol contains no personal data

\section{Competing interests}

The authors declare that they have no conflict of interest

\section{Author details}

${ }^{1}$ Charité Universitätsmedizin Berlin corporate member of Freie Universität Berlin Humboldt Universität zu Berlin, Institute of Medical Sociology Rehabilitation Science, Charitéplatz 1, 10117 Berlin, Germany. ${ }^{2}$ Technische Universität Berlin, Quality Usability Lab, Straße des 17. Juni 135, 10623 Berlin, Germany. ${ }^{3}$ German Research Centre for Artificial Intelligence (DFKI), Alt-Moabit 91c, 10559 Berlin, Germany. ${ }^{4}$ Havelland Kliniken Unternehmensgruppe, Ketziner Straße 19, 14641 Nauen, Germany.

Received: 22 October 2021 Accepted: 23 December 2021 Published online: 20 January 2022

\section{References}

1. Dixon A: The United Nations Decade of Healthy Ageing requires concerted global action. Nat Aging 2021, 1(2).

2. Collard RM, Boter H, Schoevers RA, Oude Voshaar RC. Prevalence of frailty in community-dwelling older persons: a systematic review. J Am Geriatr Soc. 2012;60(8):1487-92.
3. Xue QL. The frailty syndrome: definition and natural history. Clin Geriatr Med. 2011;27(1):1-15.

4. Bessa B, Ribeiro $O$, Coelho T. Assessing the social dimension of frailty in old age: A systematic review. Arch Gerontol Geriatr. 2018;78:101-13.

5. Travers J, Romero-Ortuno R, Bailey J, Cooney MT. Delaying and reversing frailty: a systematic review of primary care interventions. Br J Gen Pract. 2019;69(678):e61-9.

6. Baker PS. Measuring Life-Space Mobility in Community-Dwelling Older Adults. Journal of American Geriatric Societey. 2003;51(11):1610-4.

7. Hirsch JA, Winter M, Clarke P, McKay H: Generating GPS activity spaces that shed light upon the mobility habits of older adults: a descriptive analysis. Int J Health Geogr 2014, 13(51).

8. Baker PS, Bodner EV, Allman RM. Measuring Life-Space Mobility in Community-Dwelling Older Adults. J Am Geriatr Soc. 2003;51:1610-4.

9. Xue QL, Fried LP, Glass TA, Laffan A, Chaves PH. Life-space constriction, development of frailty, and the competing risk of mortality: the Women's Health And Aging Study I. Am J Epidemiol. 2008;167(2):240-8.

10. Kuspinar A, Verschoor CP, Beauchamp MK, Dushoff J, Ma J, Amster E, Bassim C, Dal Bello-Haas V, Gregory MA, Harris JE, et al. Modifiable factors related to life-space mobility in community-dwelling older adults: results from the Canadian Longitudinal Study on Aging. BMC Geriatr. 2020;20(1):35.

11. Rantanen T, Hassandra M, Pynnonen K, Siltanen S, Kokko K, Karavirta L, Kauppinen M, Sipila S, Saajanaho M, Portegijs E. The effect of individualized, theory-based counselling intervention on active aging and quality of life among older people (the AGNES intervention study). Aging Clin Exp Res. 2020;32(10):2081-90.

12. Freund AM, Baltes MM: Toward a theory of successful aging: Selection, optimization, and compensation: Hogrefe \& Huber Publishers; 2007.

13. Sniehotta FF, Schwarzer R, Scholz U, Schüz B. Action planning and coping planning for long-term lifestyle change: theory and assessment. Eur J Soc Psychol. 2005;35(4):565-76.

14. Penger S, Oswald F. A New Measure of Mobility-Related Behavioral Flexibility and Routines in Old Age. GeroPsych. 2017;30(4):153-63.

15. Walters K, Frost R, Kharicha K, Avgerinou C, Gardner B, Ricciardi F, Hunter R, Liljas A, Manthorpe J, Drennan V, et al. Home-based health promotion for older people with mild frailty: the HomeHealth intervention development and feasibility RCT. Health Technol Assess. 2017;21(73):1-128.

16. Siltanen S, Portegijs E, Pynnonen K, Hassandra M, Rantalainen T, Karavirta L, Saajanaho MJ, Rantanen T. Effects of an Individualized Active Aging Counseling Intervention on Mobility and Physical Activity: Secondary Analyses of a Randomized Controlled Trial. J Aging Health. 2020;32(10):1316-24.

17. Zijlstra W, Giannouli E. Mobility in community-dwelling older adults; what are its determinants? BMC Geriatr. 2021;21(1):228.

18. Mollenkopf H, Marcellini F, Ruoppila I, Szeman Z, Tacken M, Wahl HW. Social and behavioural science perspectives on out-of-home mobility in later life: findings from the European project MOBILATE. Eur J Ageing. 2004;1(1):45-53.

19. Dedeyne L, Deschodt M, Verschueren S, Tournoy J, Gielen E. Effects of multi-domain interventions in (pre)frail elderly on frailty, functional, and cognitive status: a systematic review. Clin Interv Aging. 2017;12:873-96.

20. Hewston P, Grenier A, Burke E, Kennedy CC, Papaioannou A. Frailty and Life-Space Mobility: Implications for Clinical Practice and Research. Occup Ther Health Care. 2021;35(1):16-24.

21. Michie S, Richardson M, Johnston M, Abraham C, Francis J, Hardeman W, Eccles MP, Cane J, Wood CE. The behavior change technique taxonomy (v1) of 93 hierarchically clustered techniques: building an international consensus for the reporting of behavior change interventions. Ann Behav Med. 2013;46(1):81-95.

22. Wurm S, Diehl M, Kornadt AE, Westerhof GJ, Wahl HW. How do views on aging affect health outcomes in adulthood and late life? Explanations for an established connection. Dev Rev. 2017;46:27-43.

23. Wolff JK, Warner LM, Ziegelmann JP, Wurm S. What do targeting positive views on ageing add to a physical activity intervention in older adults? Results from a randomised controlled trial. Psychol Health. 2014;29(8):915-32.

24. Baltes PB, Baltes MM. Erfolgreiches Altern: Mehr Jahre und mehr Leben. Bern: Huber; 1989.

25. Fillekes MP, Giannouli E, Kim EK, Zijlstra W, Weibel R. Towards a comprehensive set of GPS-based indicators reflecting the multidimensional 
nature of daily mobility for applications in health and aging research. Int J Health Geogr. 2019;18(1):17.

26. Schneider C, Rudolff C, Bauer D, Gnzález MC: Daily travel behavior: lessens from a wekk-long survey for the extraction of human mobility motifs related information. In: UrbComp'13: Proceedings on the 2nd ACM SIGKDD International Workshop on Urban Computing. 2013.

27. Smith L, Foley L, Panter J. Activity spaces in studies of the environment and physical activity: A review and synthesis of implications for causality. Health Place. 2019;58:102113.

28. Mumken SA, Gellert P, Stollwerck M, O'Sullivan JL, Kiselev J. Validation of the German Life-Space Assessment (LSA-D): cross-sectional validation study in urban and rural community-dwelling older adults. BMJ Open. 2021;11(7):e049926.

29. Craig CL, Marshall AL, Sjostrom M, Bauman AE, Booth ML, Ainsworth BE, Pratt M, Ekelund U, Yngve A, Sallis JF, et al. International physical activity questionnaire: 12 -country reliability and validity. Med Sci Sports Exerc. 2003;35(8):1381-95.

30. Sutcliffe: A new version of the Geriatric Depression Scale for Nursing and Residential Home Pupulations: The Geriatric Depression Scale (Residential) (GDS-12R). Int Psychogeriatr 2000, 12(2):173-181.

31. Yesavage JA, Sheikh JI. $9 /$ Geriatric Depression Scale (GDS). Clin Gerontol. 1968:5(1-2):165-73.

32. Morfeld M, Kirchberger I, Bullinger M. SF-36. Fragebogen zum Gesundheitszustand. Göttingen: Hogrefe; 2011.

33. Ware J, Sherbourne CD. The MOS 36-Item Short-Form Health Suvey (SF-36): Conceptual Framework and Item Selection. Med Care. 1992;30(6):473-83.

34. Fried LP, Tangen CM, Walston J, Newman AB, Hirsch C, Gottdiener J, Seeman T, Tracy R, Kop WJ, Burke G, et al. Frailty in Older Adults: Evidence for a Phenotype. J Gerontol: MEDICAL SCIENCES. 2001;56A(3):M146-56.

35. Bandeen-Roche K, Xue Q-L, Ferrucci L, Walston J, Gruralnik JM, Chaves P, Zeger SL, Fried LP. Phenotype of Frailty: Characterization in the Women's Health and Aging Studies. J Gerontol: MEDICAL SCIENCES. 2006;61A(3):262-6.

36. Lyons JG, Heeren T, Stuver SO, Fredman L. Assessing the agreement between 3-meter and 6-meter walk tests in 136 community-dwelling older adults. J Aging Health. 2015;27(4):594-605.

37. Romero-Ortuno R. The Frailty Instrument for primary care of the Survey of Health, Ageing and Retirement in Europe predicts mortality similarly to a frailty index based on comprehensive geriatric assessment. Geriatr Gerontol Int. 2013;13(2):497-504.

38. Gustafsson $\mathrm{S}$, Wilhelmson $\mathrm{K}$, Eklund $\mathrm{K}$, Gosman-Hedstrom G, Ziden L, Kronlof GH, Hojgaard B, Slinde F, Rothenberg E, Landahl S, et al. Healthpromoting interventions for persons aged 80 and older are successful in the short term-results from the randomized and three-armed Elderly Persons in the Risk Zone study. J Am Geriatr Soc. 2012;60(3):447-54.

39. Bickmore TW, Silliman RA, Nelson K, Cheng DM, Winter M, Henault L, Paasche-Orlow MK. A randomized controlled trial of an automated exercise coach for older adults. J Am Geriatr Soc. 2013;61(10):1676-83.

40. Portegijs E, Rantakokko M, Viljanen A, Sipila S, Rantanen T. Is frailty associated with life-space mobility and perceived autonomy in participation outdoors? A longitudinal study Age Ageing. 2016;45(4):550-3.

41. Wurm M, Goebel J, Taubenböck H, Wagner GG: Am Ender der Kernstadt - ein Versuch der Abgrenzung des Physischen durch das Subjektive. In: Globale Urbanisierung Perspektive aus dem All. Heidelberg; 2015.

42. Edgren J, Karinkanta S, Rantanen T, Daly R, Kujala UM, Tormakangas T, Sievanen H, Kannus P, Heinonen A, Sipila S, et al. Counselling for physical activity, life-space mobility and falls prevention in old age (COSMOS): protocol of a randomised controlled trial. BMJ Open. 2019;9(9):e029682.

43. Zhu L, Duval C, Boissy P, Montero-Odasso M, Zou G, Jog M, Speechley M Comparing GPS-Based Community Mobility Measures with Self-report Assessments in Older Adults with Parkinson's Disease. J Gerontol A Biol Sci Med Sci. 2020;75(12):2361-70.

44. Chung J, Sargent L, Brown R, Gendron T, Wheeler D. GPS Tracking Technologies to Measure Mobility-Related Behaviors in Community-Dwelling Older Adults: A Systematic Review. J Appl Gerontol. 2021;40(5):547-57.

45. Fillekes MP, Rocke C, Katana M, Weibel R. Self-reported versus GPSderived indicators of daily mobility in a sample of healthy older adults. Soc Sci Med. 2019;220:193-202.

46. Freund AM, Baltes MM: Toward a theory of successful aging: Selection, optimization, and compensation Hogrefe \& Huver Publishers; 2007.
47. Verplanken B, Orbell S. Reflections on Past Behavior: A Self-Report Index of Habit Strength1. J Appl Soc Psychol. 2003;33(6):1313-30.

48. Schwarzer R. Modeling Health Behavior Change: How to Predict and Modify the Adoption and Maintenance of Health Behaviors. Appl Psychol. 2008;57(1):1-29.

49. Schott N. Reliability and validity of the German short version of the Activities specific Balance Confidence (ABC-D6) scale in older adults. Arch Gerontol Geriatr. 2014;59(2):272-9.

50. Röthlin F, Pelikan JM, Gnahl K: Die Gesundheitskompetenz der 15-jährigen Jugendlichen in Österreich. Abschlussbericht der österreichischen Gesundheitskompetenz Jugendstudie im Auftrag des Hauptverbandes der österreichischen Sozialversicherungsträger (HVSV) In.; 2013.

51. Börsch-Supan A, Brugiavini A, Jürges H, Siegrist H, Weber G-: Health, Ageing and Retirement in Europa. First Results from the Survey of Health, Ageing and Reitrement in Europe. Mannheim: Mannheim Research Institute for the Economics of Aging (MEA); 2005.

52. Iwarsson S, Sixsmith J, Oswald F, Wahl HW, Nygren C, Sixsmith A, Szeman Z, Tomsone S: The ENABLE-AGE Project: Multi-Dimensional Methodology for European Housing Research. In. Edited by Consortium TE-AP; 2004.

53. Mahne K, Wolff JK, Simonson J: Altern im Wandel. Zwei Jahrzehnte Deutscher Alterssurvey (DEAS). Berlin: Springer VS; 2017.

54. Hughes ME, Waite LJ, Hawkley LC, Cacioppo JT. A Short Scale for Measuring Loneliness in Large Surveys. Results From Two-Population-Based Studies Res Aging. 2004;26(6):655-72.

55. de Morton NA, Davidson M, Keating JL. The de Morton Mobility Index (DEMMI): an essential health index for an ageing world. Health Qual Life Outcomes. 2008;6:63.

56. Borson S, Scanlan JM, Chen P, Ganguli M. The Mini-Cog as a Screen for Dementia: Validation in a Population-Based Sample. J Am Geriatr Soc. 2003;51:1451-4.

\section{Publisher's Note}

Springer Nature remains neutral with regard to jurisdictional claims in published maps and institutional affiliations.

Ready to submit your research? Choose BMC and benefit from:

- fast, convenient online submission

- thorough peer review by experienced researchers in your field

- rapid publication on acceptance

- support for research data, including large and complex data types

- gold Open Access which fosters wider collaboration and increased citations

- maximum visibility for your research: over 100M website views per year

At BMC, research is always in progress.

Learn more biomedcentral.com/submissions 